\title{
Desarrollo de una Escala para Valorar Conocimiento sobre Situaciones y Conductas de Riesgo de Contagio de VIH, en Jóvenes y Adultos Jóvenes en un Contexto Latinoamericano
}

\author{
Development of a Scale to Assess Knowledge on Situations and HIV-Related Risk \\ Behaviours in Youth and Young Adults in a Latin American Context
}

\author{
Geraldy Sepúlveda-Páez ${ }^{1}$, Patricio Mena-Chamorro ${ }^{2}$ y Rodrigo Ferrer-Urbina ${ }^{3}$
}

\begin{abstract}
Resumen
Este estudio presenta el desarrollo de una escala para evaluar el conocimiento sobre conductas sexuales de riesgo. La muestra se compone de 1037 jóvenes y adultos jóvenes (540 mujeres y 482 hombres) de las 5 principales ciudades del norte grande de Chile. La versión final de la escala posee 12 ítems diferenciados en 2 dimensiones: conocimiento sobre conductas de riesgo; y conocimiento sobre conductas sin riesgo. Los resultados proveen evidencias de validez basada en la estructura interna, mediante ESEM (CFI=.99; TLI=.99: RMSEA=.02), y basada en la relación con otras variables, mediante SEM, así como adecuados niveles de fiabilidad y evidencia de invarianza por sexo. La presente escala constituye un instrumento breve y con amplio respaldo psicométrico para su uso en muestras equivalentes.
\end{abstract}

Palabras clave: VIH, conocimiento sobre conductas de riesgo, escala, evidencias de validez

\begin{abstract}
This study reports the development of a scale to assess knowledge about risky sexual behaviour. The sample is composed of 1037 young people and young adults (540 women and 482 men) from the 5 main cities of the northern part of Chile. The final version of the scale has 12 items divided into 2 dimensions: knowledge about risk behaviour; and knowledge about non-risk behaviour. Results provide evidence of validity based on internal structure, through ESEM (CFI=.99;TLI=.99: RMSEA=.02), and based on the relationship with other variables, through SEM, as well as adequate levels of reliability and evidence of invariance by sex. Findings suggest that this scale is a brief instrument with broad psychometric support for use in equivalent samples.
\end{abstract}

Keywords: HIV, risk behavior, scale, validity evidence

\footnotetext{
${ }_{1}^{1}$ Psicóloga, Universidad de Tarapacá, Chile. Tel.: +569 62793001. Correo: geraaldy@ gmail.com. ORCID https://orcid.org/0000-0002-4693-7937

${ }^{2}$ Psicólogo, Universidad de Tarapacá, Chile. Tel.: + 569 65316280. Correo: mena_pato@live.cl ORCID https://orcid.org/0000-0001-7397-2845

${ }^{3}$ Escuela de Psicología y Filosofía, Universidad de Tarapacá, 18 de septiembre \#2222, Arica, Chile. Tel.: +56582205640. Correo: rferrer@academicos.uta.cl. ORCID https://orcid.org/0000-0001-5407-3158
} 


\section{Introducción}

El Virus de inmunodeficiencia humana (en adelante, VIH) y su fase activa, el Síndrome de inmunodeficiencia adquirida (en adelante, SIDA) es un problema de salud pública que afecta a millones de personas en todo el mundo (Edo \& Ballester, 2006; Harding \& Molloy, 2008; Organización Mundial de la Salud [OMS], 2018; Programa Conjunto de las Naciones Unidas sobre el VIH/SIDA [ONUSIDA], 2020).

En Chile, la tasa de casos confirmados de VIH/SIDA alcanzo las 43386 personas, lo que equivale a un aproximado de 240 personas, cada 100000 habitantes, viviendo con VIH/SIDA (Cáceres-Burton, 2019). Con excepción de la Región Metropolitana (i.e. la cual contiene la capital nacional, concentra un tercio de los habitantes del país y ocupa el tercer lugar entre las regiones con mayores tasas), las tres regiones del Norte Grande de Chile poseen las mayores tasas de nuevos diagnósticos de VIH/SIDA, observándose, por ejemplo, que la tasa de nuevas notificaciones de VIH del año 2017, en la región de Arica y Parinacota $(50,6$ cada $100000 \mathrm{Hb})$ fue 2,8 veces la tasa nacional (17,9 cada $100000 \mathrm{Hb})$ (Ministerio de Salud de Chile, [MINSAL], 2017).

Debido a la magnitud de esta problemática, a nivel mundial y local, se han desarrollado múltiples esfuerzos para disminuir el crecimiento de esta pandemia, incluyendo programas de prevención, atención y seguimiento (Fisher \& Fisher, 2000; MINSAL, 2018; OMS, 2018). Sin embargo, a pesar de los avances, el número de personas contagiadas continúa en aumento (ONUSIDA, 2019), siendo la población de mayor riesgo los adultos entre 20 a 39 años, quienes presentan la tasa más alta en Chile y el mundo (Cáceres-Burton, 2019; Instituto de Salud Pública, [ISP], 2016; ONUSIDA, 2018). Históricamente, el VIH se ha concentrado en hombres que tienen sexo con hombres (OMS, 2018), no obstante, en los últimos años, se ha observado un mayor crecimiento en la proporción de hombres y mujeres que declaran tener, únicamente, relaciones heterosexuales (Dworkin, 2015; Santos, Couto, Mathias, \& Grangeiro, 2020).

El hecho de que los nuevos contagios se focalicen en jóvenes y adultos jóvenes, se ha explicado por la conjunción de dos aspectos claves en la transmisión de VIH: una vida sexual activa y una mayor tendencia a realizar conductas sexuales de riesgo (Calatrava, López-del Burgo, \& de Irala, 2012; Herdoiza-Arroyo \& Chóliz, 2019; Pérez-Morente et al., 2017).

Las conductas sexuales de riesgo refieren a la eventual exposición del individuo a situaciones o comportamientos que puedan causar daños en su salud sexual o en la salud de otros (EspadaSánchez, Quiles- Sebastían, \& Méndez Carrillo, 2003; Spencer, Doull, \& Shoveller, 2014). Entre las principales conductas sexuales de riesgo relacionadas con la transmisión de VIH, se identifican: relaciones sexuales con uso inadecuado de preservativo; sexo bajo la influencia del consumo de alcohol y/o drogas; y multiplicidad de parejas sexuales (BouniotEscobar, Muñoz-Vigueras, Norambuena-Vergara, Pinto-Ulloa, \& Muñoz-Pareja, 2017; Spindola et al., 2020).

Existen una serie de factores que se han relacionado con las conductas sexuales de riesgo, ya sea como factores protectores o de riesgo, tales como: personalidad, autoestima, riesgo percibido, actitudes sexuales y asertividad sexual (e.g. Grov, Parsons, \& Bimbi, 2010; Prata, Morris, Mazive, Vahidnia, \& Stehr, 2006; Santos-Iglesias, VallejoMedina, \& Sierra, 2014; De Wilde, Casini, Wollast, \& Demoulin, 2020; Zetterqvist et al., 2018).

No obstante, dentro de estos factores, los que han recibido mayor atención en las campañas de salud, son aquellos referidos al conocimiento de los comportamientos y situaciones de riesgo (e.g. Janulis, Newcomb, Sullivan, \& Mustanski, 2017; Velo-Higueras et al., 2018).

El conocimiento sobre las conductas sexuales de riesgo refiere al grado de información sobre aquellas acciones y/o situaciones potencialmente infecciosas y su diferenciación de aquellas que no lo son (Bahamón, Vianchá, \&Tobos, 2014). En general, el conocimiento sobre situaciones y conductas sexuales de riesgo se ha considerado como uno de los determinantes claves para la generación de un cambio en el comportamiento y prevención de nuevas enfermedades (Carey \& Lewis, 1999; Collado, Loya, \& Yi, 2015; Fuentes, Teva, \& Bermúdez, 2016; Jemmott, Jemmott, Fong, \& Morales, 2010), siendo señalado en distintos modelos teóricos, como el Modelo de 
Creencias en Salud, (Rosenstock, Strecher, \& Becker, 1988), Teoría de Aprendizaje Social (Bandura, Freeman, \& Lightsey, 1999) y el Modelo información, motivación-habilidades (Fisher \& Fisher, 2002), como prerrequisito o factor influyente en los cambios de comportamientos. Este aspecto se ha relacionado con un mayor uso del condón y con actividades coitales de menor riesgo (e.g. Villaseñor-Sierra, Caballero-Hoyos, Hidalgo san Martín, \& SantosPreciado, 2003; Villar-Loubet et al., 2013), un menor estigma y actitud favorable hacia quienes presentan una ITS o VIH (Huy et al., 2016).

Sin embargo, pese a que las campañas de prevención, realizadas en Chile, se han focalizado en la entrega de información, promoviendo la realización de pruebas y uso del condón (MINSAL, 2017), los resultados no son alentadores, ya que no se ha evidenciado una reducción en la progresividad del virus (CáceresBurton, 2019; [ISP], 2016). Existe la posibilidad que los efectos de estas campañas no sean los esperados, ya sea porque el conocimiento es, por sí solo, una variable insuficiente (Mittal, Senn, \& Carey, 2012) o porque las campañas no han sido efectivas en el incremento de conocimiento (Dodds \& García del Río, 2017; Ugalde et al., 2020). Por tanto, para poder desarrollar una valoración adecuada de las campañas preventivas, es necesario disponer de instrumentos de medida adecuados, que permitan identificar los niveles de conocimiento poblacionales y los efectos de las diversas campañas de prevención (Tangarife \& Arias, 2015).

En la literatura existen distintas evaluaciones que miden el conocimiento sobre conductas sexuales de riesgo de infección de VIH, tales como: (1) The HIV Knowledge Questionnaire, 1 dimensión, 45 ítems (HIV-KQ; Carey, MorrisonBeedy, \& Johnson, 1997); su versión breve 18 ítems (HIV-KQ 18; Carey \& Schroder, 2002); (2) AIDS Questionaire, 5 dimensiones, 164 ítems (Paniagua, et al.,1994); (3) Questionnaire to Measure Knowledge of Sexually Transmitted Diseases, 2 dimensiones, 27 ítems (STD-KQ; Jaworski \& Carey, 2007); (4) the HIV/AIDS Knowledge Scale, 3 dimensiones, 10 ítems (HIVKS; Espada et al., 2009); (5) AIDS Prevention Questionnaire, 5 dimensiones, 44 ítems (GilLlario, Ruiz-Palomino, Morell-Mengual,
Giménez-García, \& Ballester-Arnal, 2018). Incluyendo la adaptación al español de la escala de VIH- 65 desarrollada Bermúdez, Sánchez y Buela-Casal (2005) (como se citó en Pineda, Rodríguez, \& Orbegozo, 2013).

$\mathrm{Si}$ bien, la mayoría de ellos cuenta con evidencias psicométricas (Carey \& Schroder, 2002; Espada et al., 2009), gran parte de estos han sido elaborados en idiomas distintos del español, no presentan adaptaciones para su aplicación en población sudamericana (e.g Janulis et al., 2018; Pineda-Roa, Campos-Areas,2018; Velo-Higueras., et al 2018) y, las adaptaciones idiomáticas disponibles, corresponden a desarrollos multidimensionales que no refieren específicamente al conocimiento (Bermúdez et al., 2005; Gil-Llario et al., 2018; Paniagua, et al., 1994). Por tanto, cuando se realizan estudios de conocimiento de conductas de riesgo en contextos sudameamericanos, predomina el uso de elaboraciones ad-hoc, entrevistas y/o instrumentos carentes de evidencias de validez (e.g. OrdoñezSánchez, Real Cotto, Gallardo León, Alvarado Franco, \& Roby Arias, 2017; Villaseñor-Sierra et al., 2003).

El presente estudio tiene por propósito desarrollar una escala para medir el conocimiento sobre situaciones y conductas de riesgo de contagio de VIH, incluyendo la capacidad para diferenciar aquellas que no constituyen riesgo, en jóvenes y adultos jóvenes del Norte Grande de Chile.

\section{Método}

\section{Diseño y participantes}

La presente investigación corresponde a un estudio transversal, ex-post facto, de tipo instrumental.

El muestreo fue no probabilístico, de tipo tiempo-espacio, ya que esta estrategia ha evidenciado ser de utilidad en estudios de VIH/SIDA (Semaan, Lauby, \& Liebman, 2002). Esta investigación consideró como criterio de inclusión residir en las ciudades del norte grande de Chile y tener 18 años o más, una vez recolectado los datos, se excluyeron los casos que contestaron menos de un $80 \%$ del cuestionario.

La muestra fue 1037 jóvenes y adultos de 18 a 35 años, residentes en las ciudades de Arica $(21,2 \% ; n=220)$, Iquique $(14,0 \% ; n=145)$, Alto 
Hospicio (10,5\%; $\mathrm{n}=109)$, Antofagasta $(37,3 \%$; $\mathrm{n}=387)$ y Calama $(17,0 \% ; \mathrm{n}=176)$, siendo un $47,1 \% \quad(\mathrm{n}=482)$ hombres, un 52,7\% $(\mathrm{n}=540)$ mujeres y un $0,2 \%(n=2)$ intersexuales, con una edad media de 22 años (DT=5,3). Del total de los encuestados un $87,2 \% \quad(n=904)$ declaró estar soltero, un $82,3 \%(n=853)$ se autoidentificó como heterosexual, un $4,1 \%(n=42)$ declaró haber sido diagnosticado con una o más ITS y un $0,6 \%(n=6)$ declaró ser VIH positivo.

\section{Instrumentos}

Escala de conocimiento sobre situaciones y conductas de riesgo de contagio de VIH: cuestionario cuyo propósito es valorar el conocimiento sobre conductas y situaciones de riesgo de contagio de VIH. La escala final consta de 12 ítems, destinados a evaluar 2 dimensiones: 1) Conocimiento sobre conductas de riesgo (en adelante, CR; ítems=5); 2) Conocimiento sobre conductas sin riesgo (en adelante, CSR; ítems=7). La inclusión de la dimensión sobre reconocimiento de conductas sin riesgo tuvo la finalidad de generar un punto de contraste, de modo de evitar confundir efectos de aquiescencia, $\mathrm{u}$ otros sesgos de respuesta, con aciertos. Las preguntas de la escala de conocimiento son afirmaciones conductuales donde algunas refieren a conductas sexuales que constituyen riesgos reales de transmisión y otros que refieren a interacciones con personas portadoras de VIH que no constituyen un riesgo de contagio. La escala presenta una consigna general para ambas dimensiones, donde se señalan las dos opciones de respuesta (es un riesgo y no es un riesgo), asignándose un acierto (1) cuando la clasificación es adecuada y un error ( 0 ) cuando la clasificación es inadecuada. La puntuación es de 0 a 5 para la dimensión de conocimiento de conductas con riesgo y de 0 a 7 para la dimensión de conocimiento de conductas sin riesgo.

Inicialmente se recopiló un listado de conductas a través de una revisión bibliográfica sobre vías de transmisión y prejuicios comunes de $\mathrm{VIH}$, las cuales fueron complementadas $\mathrm{o}$ corregidas por 2 profesionales del área de salud sexual y reproductiva. Con las conductas recopiladas, se crearon 40 ítems diferenciales para evaluar el conocimiento sobre situaciones y conductas de riesgo de contagio de VIH, (26 ítems, que representaban un riesgo y 14 ítems sin riesgo). El instrumento inicial fue sometido a una depuración de redacción y contenido mediante jueces expertos ( 3 psicólogos con experiencia en psicometría y 2 profesionales del área de la salud), de esta revisión se conservaron 22 ítems, con los que se realizó una aplicación piloto online en jóvenes universitarios $(n=524)$, no incluida en el presente estudio. Una vez finalizada, se realizó una depuración basada la consistencia interna y coeficientes de fiabilidad (se eliminaron aquellos ítems con saturaciones factoriales débiles $(\lambda<.3)$ y/o que presentaban redundancias) (Cho \& Kim, 2014). Finalmente, se conservaron 16 ítems, los cuales fueron aplicados en el presente estudio.

Además, se recabó información sociodemográfica de los encuestados (sexo, edad, lugar de residencia y nivel educacional). La variable nivel educacional tenía 4 opciones respuesta (estudios básicos, estudios medios, estudios técnicos y estudios superiores) y fue utilizada para obtener otras evidencias de validez.

\section{Procedimiento}

El instrumento aplicado formo parte de un cuestionario mayor, que tenía como propósito medir distintas variables que afectan las conductas sexuales. La aplicación del cuestionario se realizó de forma presencial (papel y lápiz), en organizaciones públicas y sociales, en áreas de recreación y tiempos libres en las 5 principales ciudades del Norte Grande de Chile (i.e. Arica, Iquique, Alto Hospicio, Calama y Antofagasta). Junto al cuestionario se proporcionó un consentimiento informado, donde se establecían los objetivos de la investigación, la confidencialidad, anonimato y los derechos del participante. El anonimato fue salvaguardado por la devolución del cuestionario en un sobre sellado, sin ninguna forma de identificación personal. El procedimiento de respuesta tuvo una duración de 15 minutos. Tanto el instrumento como el procedimiento fueron conocidos y aprobados por el comité de ética científico de la Universidad de Tarapacá.

\section{Análisis estadístico}

Inicialmente, para evaluar la adecuación de los ítems se realizó un análisis de ítems para el modelo inicial (16 ítems), en base a la correlación 
Tabla 1. Indicadores de ajuste global, modelo inicial y depurado de la escala de conocimiento sobre situaciones y conductas de riesgo de contagio de VIH

\begin{tabular}{|c|c|c|c|c|c|c|c|c|c|c|}
\hline \multirow{2}{*}{ Modelo } & \multirow{2}{*}{$\mathrm{N}^{\circ} \mathrm{Par}$} & \multirow{2}{*}{$\chi^{2}$} & \multirow{2}{*}{ DF } & \multirow{2}{*}{$\mathrm{P}$} & \multirow{2}{*}{ CFI } & \multirow{2}{*}{ TLI } & \multirow{2}{*}{ RMSEA } & \multicolumn{2}{|c|}{ RMSEA $90 \%$ CI } & \multirow{2}{*}{ SRMR } \\
\hline & & & & & & & & LO & HI & \\
\hline $\begin{array}{l}\text { ESEM } \\
\text { (Ítems=13) }\end{array}$ & 38 & 200.590 & 53 & .00 & .970 & .956 & .052 & .04 & .06 & .052 \\
\hline $\begin{array}{l}\text { ESEM } \\
\text { (Ítems=12) }\end{array}$ & 35 & 182.559 & 43 & .00 & .995 & .992 & .025 & .01 & .03 & .031 \\
\hline $\begin{array}{l}\text { CFA } \\
\text { (Ítem=12) }\end{array}$ & 25 & 238.939 & 53 & .00 & .961 & .952 & .059 & .05 & .06 & .085 \\
\hline
\end{tabular}

Nota. $\mathrm{x}^{2}=$ Chi cuadrado; $\mathrm{DF}=$ grados de libertad; $P=$ significación; $\mathrm{CFI}=$ índice de ajuste comparativo; TLI=índice de Tucker Lewis RMSEA=error de aproximación; $\mathrm{CI}=$ intervalo de confianza; SRMR=raíz cuadrada media estandarizada residual.

ítem total corregida, descartándose aquellos con índices de homogeneidad corregidos pequeños (<.2), y los análisis de correlación inter ítem, descartándose aquellos ítems que presentaban redundancias de consideración (>.5), conservándose 13 ítems (Para más detalle sobre estos análisis ver anexo 6,7 y 8).

Con el modelo de 13 ítems, se realizó un modelo de ecuaciones estructurales exploratorios (ESEM) con rotación GEOMIN (Asparouhouv \& Muthén, 2009), a partir de la matriz de variables policóricas, siguiendo recomendaciones sobre el tratamiento factorial de variables ordinales (Garrido, Abad, \& Ponsoda, 2011), utilizando el método de estimación mínimos cuadrados ponderados robustos (WLSMV), el cual es robusto con variables discretas no normales (Asparouhov \& Muthen, 2007). Además, con la finalidad de corroborar que las saturaciones factoriales cruzadas no generaran impactos relevantes en el modelo, se realizó un análisis factorial confirmatorio (AFC), utilizando la matriz de variables policóricas y el método de estimación mínimos cuadrados ponderados robustos (WLSMV) recomendados por Ledesma, Ferrando y Tosi (2019). El ajuste general del modelo se evaluó acorde los puntos de cortes recomendando por Schreiber (2017) (e.g. CFI> .95; TLI> .95; RMSEA <.06). Como tercera etapa se estimó la fiabilidad para cada dimensión, a través del Alpha de Cronbach ordinal y omega jerárquico de McDonald (Gadermann, Guhn, \& Zumbo, 2012). Con el propósito de evaluar la equivalencia de las puntuaciones entre personas de diferentes sexos, se realizaron pruebas de invarianza, utilizando como criterio de invarianza la disminución de CFI (>.005) y el aumento de RMSEA (<.010) (Chen, 2007).

Finalmente, la evidencia de validez basada en la convergencia, se calculó a través de un modelo ESEM, entre las dimensiones de la escala de conocimiento y el nivel educacional, utilizando el método de estimación WLSMV y la matriz de correlaciones policóricas. Los análisis se realizaron mediante el programa Mplus (8.0) y Jamovi versión (9.0).

\section{Resultados}

La Tabla 1 presenta el ajuste de los modelos de medida inicial y depurado. Al observar el modelo inicial (13 ítems), los indicadores de medida reflejan un buen ajuste de acuerdo a los estándares recomendados en la literatura (Schreiber, 2017) (ver anexo 5). Sin embargo, al observar los índices de modificación, se identificó una covariación relevante entre 2 ítems de dimensiones distintas, optándose por generar un segundo modelo (12 ítems), cuyos indicadores de ajuste tanto en su versión ESEM como CFA, indica que el modelo final es una buena representación poblacional de las relaciones observadas (e.g. CFI>.95; TLI>.95; RMSEA<.06; Schreiber, 2017).

La escala depurada se compone de 12 ítems, diferenciados en 2 dimensiones; conocimiento sobre conductas de riesgo (5 ítems) y Conocimiento sobre conductas sin riesgo (7 ítems), cuyas saturaciones factoriales y estimaciones de fiabilidad (Alpha de Cronbach y coeficiente Omega) se presentan en la Tabla 2. 
Tabla 2. Cargas factoriales y estimaciones de fiabilidad de cada dimensión de los modelos de medida depurados

\begin{tabular}{|c|c|c|}
\hline & $\begin{array}{c}\text { Conocimiento } \\
\text { conductas de riesgo }\end{array}$ & $\begin{array}{c}\text { Conocimiento } \\
\text { conductas sin riesgo }\end{array}$ \\
\hline Practicar sexo oral sin preservativo a una pareja estable. & .64 & -.03 \\
\hline Realizar roces genitales externos sin preservativos. & .53 & -.12 \\
\hline Tener relaciones sexuales anales con una pareja estable, sin usar preservativo. & .70 & .00 \\
\hline Tener solo una relación sexual sin preservativo. & .58 & .27 \\
\hline $\begin{array}{l}\text { Colocarse el preservativo después de haber iniciado la penetración, pero antes del } \\
\text { orgasmo. }\end{array}$ & .64 & .34 \\
\hline Bañarse en piscinas con personas con VIH/SIDA. & .04 & .80 \\
\hline Tener algún tipo de contacto piel con piel con alguna persona portadora de VIH/SIDA. & .04 & .74 \\
\hline $\begin{array}{l}\text { Comer en las mismas vajillas o compartir utensilios con una persona con alguna ITS y/o } \\
\text { VIH/SIDA. }\end{array}$ & .04 & .77 \\
\hline Usar el mismo baño con personas con VIH/SIDA & -.04 & .84 \\
\hline Vivir con personas con VIH/SIDA. & -.03 & .91 \\
\hline Realizar cualquier actividad bajo el mismo techo con personas con VIH/SIDA. & -.08 & .91 \\
\hline Acariciar animales que convivan con personas portadoras de VIH/SIDA. & .01 & .77 \\
\hline Alpha de Cronbach & .74 & .92 \\
\hline Coeficiente Omega & .80 & .93 \\
\hline
\end{tabular}

Tabla 3. Contraste de Invarianza de medida

\begin{tabular}{lccccccccccc}
\hline & $\mathrm{N} p a r$ & $\chi^{2}$ & $\mathrm{DF}$ & $\mathrm{P}$ & $\mathrm{CFI}$ & RMSEA & $\Delta_{\chi 2}$ & $\Delta_{\mathrm{gl}}$ & $\mathrm{P}_{\Delta}$ & $\Delta_{\text {CFI }}$ & $\Delta_{\text {RMSEA }}$ \\
\hline Configuración & 74 & 268.312 & 106 & .000 & .937 & .055 & --- & --- & --- & --- & --- \\
Métrico & 64 & 266.331 & 116 & .000 & .942 & .051 & 13.614 & 20 & .849 & -.005 & .004 \\
Escalar & 54 & 281.926 & 126 & .000 & .940 & .050 & 15.595 & 10 & .111 & .002 & .001 \\
\hline
\end{tabular}

Nota. $\mathrm{X}^{2}$ : chi-cuadrado; gl: grados de libertad; $\mathrm{p}$ : significancia; $\Delta \mathrm{X} 2$ : cambio en chi-cuadrado; $\Delta \mathrm{gl}$ : cambio en los grados de libertad; $\Delta \mathrm{p}$ : cambio de significación; $\Delta_{\mathrm{CFI}}$ cambio en el índice de ajuste comparativo; $\Delta_{\mathrm{RMSEA}}$ : cambio en el error cuadrático medio de aproximación.

Las saturaciones factoriales indican que todos los ítems son representaciones adecuadas $(\lambda>.5)$ de sus dimensiones, con bajos niveles de saturaciones cruzadas $(\lambda \leq .3)$. En el caso de la dimensión conocimiento sobre conductas de riesgo, las estimaciones de fiabilidad son óptimas para el coeficiente omega ( $\omega>.80$; Cho \& Kim, 2014) y, al menos, suficientes para Alpha de Cronbach $(\alpha>$.70; Cho \& Kim, 2014), mientras que en la dimensión conocimiento de conductas sin riesgo, la fiabilidad estimada es óptima para ambos coeficientes $(\omega>.80 ; \alpha>.80$; Cho \& Kim, 2014).

La Tabla 3 presenta el análisis de invarianza entre mujeres y hombres de la escala final (12 ítems), se puede observar que los diferenciales de CFI y RMSEA, no evidencian cambios de ajuste relevantes (Chen, 2007) al fijar la igualdad en las saturaciones factoriales entre grupos. Por lo tanto, las cargas factoriales tendrían equivalencia entre hombres y mujeres.
La Tabla 4 presenta las relaciones entre las dimensiones de la escala de conocimiento sobre situaciones y conductas de riesgo de contagio de VIH y el nivel educacional. El modelo propuesto, presenta un buen ajuste (CFI=.960; TLI=.951; RMSEA=.052).

En términos generales, el nivel educacional presenta efectos estadísticamente significativos en ambas dimensiones (CCR Y CSR). Se observa un efecto directo leve entre el nivel educacional y el CCR. Mientras que entre el nivel educacional y CSR se observa un efecto directo moderado.

Tabla 4. Relaciones entre las dimensiones de la escala de conocimiento y nivel educacional

\begin{tabular}{ccc}
\hline & $\mathrm{CCR}$ & $\mathrm{CSR}$ \\
\hline Nivel educacional & $.249 *$ & $.343 *$ \\
\hline$* p<.05 ; \mathrm{CR}=$ conductas de riesgo; $\mathrm{CSR}=$ conductas sin riesgo.
\end{tabular}




\section{Discusión}

El objetivo del estudio fue elaborar y proporcionar evidencias de validez de una escala para medir el conocimiento sobre situaciones y conductas de riesgo de contagio de VIH, en jóvenes y adultos jóvenes en un contexto latinoamericano.

Para dar cumplimiento a este objetivo, se analizó la estructura interna del instrumento mediante el análisis de ítems, análisis de fiabilidad, modelos ESEM, análisis de invarianza y convergencia.

Los resultados del modelo final, la magnitud de las saturaciones factoriales y la inexistencia de saturaciones cruzadas relevantes, constituyen evidencias que respaldan la estructura propuesta. Las estimaciones de fiabilidad de cada dimensión (e.i, conocimiento de conductas de riesgo y conocimiento de conductas sin riesgo), presentan niveles adecuados o al menos suficientes de consistencia interna. Además, los resultados del análisis de invariancia, permiten sostener invariancia métrica y escalar (Chen, 2007), lo que hace posible aplicar e interpretar la escala de un mismo modo, tanto en hombres como en mujeres, ya que las saturaciones factoriales son equivalentes entre los grupos.

Finalmente, respecto a la evidencia de validez basada en la relación con otras variables, la escala de conocimiento sobre situaciones y conductas de riesgo de contagio de VIH y el nivel educacional presentaron asociaciones estadísticamente significativas, lo cual concuerda con otros estudios (Chirwa, 2019; Stutts, Robinson, Witt, \& Terrell, 2020 ) donde se ha evidenciado que nivel educacional influye en el conocimiento que se tenga sobre el VIH y las conductas que puedan implicar riesgo de contagio.

A pesar de la evidencia observada, existen algunas restricciones, principalmente por el carácter no probabilístico de la muestra y, sobre todo, su acotación a un conjunto de regiones particulares. En este escenario, es recomendable ampliar el uso de la escala a nuevas regiones y contextos, para evaluar su adecuación a ellos y aumentar sus evidencias de validez. Por otra parte, ya que a mayoría de los encuestados se identificó como heterosexual es necesario replicar los análisis en poblaciones que pueden presentar particularidades en su exposición al riesgo (e.g. hombres que tienen sexo con hombres; personas transgenero; trabajadoras sexuales), y así incrementar la capacidad de generalización del uso de la escala.

$\mathrm{Si}$ bien existen instrumentos que miden el conocimiento sobre conductas sexuales, infecciones de trasmisión sexual, o VIH. Los cuales están disponibles y han sido utilizados en contextos latinoamericanos (Jaworski \& Carey, 2007; Ugalde et al., 2020). La presente escala posee la ventaja de ser desarrollada desde la propia particularidad cultural, contar con evidencias de fiabilidad y validez robusta y, sobre todo, al focalizarse exclusivamente en el conocimiento sobre conductas y situaciones de riesgo de contagio de VIH, la ventaja de constituir una escala de rápida aplicación y fácil integración en cualquier batería de evaluación (con una aplicación aproximada de un par de minutos). Por lo tanto, la presente escala constituye un aporte practico, científicamente respaldado, para estudios de tamizaje, correlacionales e, inclusive, la evaluación de campañas de prevención.

\section{Referencias}

Asparouhouv, T., \& Muthén, B.O. (2007). Wald test of mean equality for potential latent class predictors in mixture modeling. Recuperado de:

http://www.statmodel.com/download/MeanTe st1.pdf

Asparouhov, T., \& Muthén, B. (2009). Exploratory structural equation modeling. Structural equation modeling: $A$ Multidisciplinary Journal, 16(3), 397-438. doi.org/10.1080/10705510903008204

Bahamón, M., Vianchá, M., \& Tobos, A. (2014). Prácticas y conductas sexuales de riesgo en jóvenes: Una perspectiva de género. Psicología Desde El Caribe, 31(2), 327-343. doi.org/10.14482

Bandura, A., Freeman, W. H., \& Lightsey, R. (1999). Self-Efficacy: The Exercise of Control.

Bouniot-Escobar, S. V., Muñoz-Vigueras, C. A., Norambuena-Vergara, N. R. M., Pinto-Ulloa, C. F., \& Muñoz-Pareja, M. A. (2017). 
Prevalencia de conductas sexuales de riesgo en estudiantes de primer año de pregrado de la universidad San Sebastián, Concepción, Chile, 2016: estudio descriptivo. Revista Colombiana de Obstetricia y Ginecología, 68(3), 176-185. doi:/10.18597/rcog.2799

Cáceres-Burton, Karen. (2019). Informe: Situación epidemiológica de las infecciones de transmisión sexual en Chile, 2017. Revista Chilena de Infectología, 36(2), 221-233. doi.org/10.4067/S0716-10182019000200221

Calatrava, M., López-Del Burgo, C., \& de Irala, J. (2012). Factores de riesgo relacionados con la salud sexual en los jóvenes europeos. Medicina Clínica, 138(12), 534-540. doi:10.1016/j.medcli.2011.07.020

Carey, M. P., \& Lewis, B. P. (1999). Motivational strategies can enhance HIV risk reduction programs. AIDS and Behavior, 3(4), 269-276. doi.org/10.1023/A:1025429216459

Carey, M. P., Morrison-Beedy, D., \& Johnson, B. T. (1997). The HIV-Knowledge Questionnaire: Development and evaluation of a reliable, valid, and practical selfadministered questionnaire. AIDS and Behavior, 1(1), 61-74. doi.org/10.1023/A:1026218005943

Carey, M. P., \& Schroder, K. E. (2002). Development and psychometric evaluation of the brief HIV Knowledge Questionnaire. AIDS Education and Prevention, 14(2), 172182. doi.org/10.1521/aeap.14.2.172.23902

Chen, F. F. (2007). Sensitivity of Goodness of Fit Indexes to Lack of Measurement Invariance. Structural Equation Modeling: A Multidisciplinary Journal, 14(3), 464-504. doi.org/10.1080/10705510701301834

Cho, E., \& Kim, S. (2014). Cronbach's coefficient alpha: Well-known but poorly understood. Organizational Research Methods, 18(2), 207-230 doi.org/10.1177/1094428114555994

Collado, A., Loya, J. M., \& Yi, R. (2015). The interaction of HIV knowledge, perceived risk, and sex differences on risky sex. International Journal of Sexual Health, 27(4), 418-428. doi.org/10.1080/19317611.2015.1031312

Dodds, T., \& García del Río, F. (2017). Evaluando la relación entre el consumo televisivo y las actitudes hacia personas viviendo con VIH/SIDA en Chile.
Investigación y Desarrollo, 25(1), 6-22. doi.org/10.14482/indes.25.1.10225

Dworkin, S. L. (2015). Men at risk: Masculinity, heterosexuality, and HIV prevention. New York: NYU Press.

Edo, M., \& Ballester, R. (2006). Estado emocional y conducta de enfermedad en pacientes con VIH/SIDA y enfermos oncológicos. Revista de Psicopatología y Psicología Clínica, 11(2), 79-90. doi:10.5944/rppc.vol.11.num.2.2006.4019

Espada, J. P., Huedo-Medina, T. B., Orgilés, M., Secades, R., Ballester, R., \& Remor, E. (2009). Psychometric properties of the HIV/AIDS Knowledge Scale for Spanish adolescents (HIV-KS). Salud y Drogas, 9(2), 149-164.

Espada-Sánchez, J. P., Quiles-Sebastián, M. J., \& Méndez-Carrillo, J. M. (2003). Conductas sexuales de riesgo y prevención del Sida en la adolescencia. Papeles del Psicólogo, 24(85), 1-15.

Fisher, J. D., \& Fisher, W. A. (2000). Theoretical approaches to individual-level change in HIV risk behavior. In Handbook of HIV prevention (pp. 3-55). Springer, Boston, MA.

Fisher, J. D., \& Fisher, W. A. (2002). The information-motivation-behavioral skills model. Emerging theories in health promotion practice and research: Strategies for improving public health, 1, 40-70.

Fuentes, A. Z., Teva, I., \& Bermúdez, M. P. (2016). Conocimiento y Fuentes de Información sobre las ITS/VIH, Comunicación sobre Sexo y Actitud Hacia el Uso del Preservativo en Adolescentes y Padres/Madres Salvadoreños. Revista Iberoamericana de Diagnóstico y Evaluación - e Avaliação Psicológica, 3(45), 97-107. doi.org/10.21865/RIDEP45.3.08

Gadermann, A. M., Guhn, M., \& Zumbo, B. D. (2012). Estimating ordinal reliability for Likerttype and ordinal item response data: A conceptual, empirical, and practical guide. Practical Assessment, Research and Evaluation, 7(3), 1-13. doi.org/10.7275/n560j767

Garrido, L., Abad, F., \& Ponsoda, V. (2011). Performance of velicer's minimum average partial factor retention method with 
categorical variables. Educational and Psychological Measurement, vol. 71(3), 551570. doi:10.1177/0013164410389489

Gil-Llario, M.D, Ruiz-Palomino, E., MorellMengual, V., Giménez-García, C., \& Ballester-Arnal, R. (2018). Validación del Cuestionario de prevención del SIDA: Un breve instrumento de autoinforme para evaluar el riesgo de infección por el VIH y orientar el cambio de comportamiento. AIDS Behav 23 (1), 272-282. doi.org/10.1007/s10461-018-2224-0

Grov, C., Parsons, J. T., \& Bimbi, D. S. (2010). Sexual compulsivity and sexual risk in gay and bisexual men. Archives of sexual behavior, 39(4), 940-949. doi.org/10.1007/s10508-009-9483-9

Harding, R., \& Molloy, T. (2008). Positive futures? The impact of HIV infection on achieving health, wealth and future planning. AIDS Care, 20(5), 565-570. doi: 10.1080/09540120701867222

Herdoiza-Arroyo, P., \& Choliz, M. (2019). Impulsivity in Adolescence: Use of a Short Version of the UPPS Questionnaire in a Sample of Latin American and Spanish Youths. Revista Iberoamericana de Diagnóstico y Evaluación - e Avaliação Psicológica, 1(50), 123-135. doi.org/10.21865/RIDEP50.1.10

Huy, N. V., Lee, H.-Y., Nam, Y.-S., Tien, N. V., Huong, T. T. G., \& Hoat, L. N. (2016). Secular trends in HIV knowledge and attitudes among Vietnamese women based on the Multiple Indicator Cluster Surveys, 2000, 2006, and 2011: what do we know and what should we do to protect them? Global Health Action, 9(1), 29247.

doi:10.3402/gha.v9.29247

Instituto de Salud Pública. (2016). Resultados confirmación de infección por VIH en Chile, 2010-2015 (pp. 3-12). Santiago. Recuperado de

http://www.ispch.cl/sites/default/files/Boletin VIH-15112017A.pdf.

Janulis, P., Newcomb, M.E, Sullivan, P., \& Mustanski, B. (2018). Evaluating HIV knowledge questionnaires among men who have sex with Men: A multi-study item response theory analysis. Archives of Sexual Behavior 47 (107-119)

doi.org/10.1007/s10508-016-0910-4

Jaworski, B. C., \& Carey, M. P. (2007). Development and psychometric evaluation of a self-administered questionnaire to measure knowledge of sexually transmitted diseases. AIDS and Behavior, 11(4), 557-574. doi.org/10.1007/s10461-006-9168-5

Jemmott III, J. B., Jemmott, L. S., Fong, G. T., \& Morales, K. H. (2010). Effectiveness of an HIV/STD risk-reduction intervention for adolescents when implemented by community-based organizations: A clusterrandomized controlled trial. American Journal of Public Health, 100(4), 720-726. doi.org/10.2105/AJPH.2008.140657

Ledesma, R. D., Ferrando, P. J., \& Tosi, J. D. (2019). Uso del Análisis Factorial Exploratorio en RIDEP. Recomendaciones para Autores y Revisores. Revista Iberoamericana de Diagnóstico y Evaluación - e Avaliação Psicológica, 52(3), 173-180. doi.org/10.21865/RIDEP52.3.13

Lozano, L. M., García-Cueto, E., \& Muñiz, J. (2008). Effect of the number of response categories on the reliability and validity of rating scales. Methodology, 4(2), 73-79. doi:10.1027/1614-2241.4.2.73

Ministerio de Salud República de Chile, Unidad de Epidemiología (2017). Informe Epidemiológico Preliminar de las Infecciones de Transmisión Sexual en Chile hasta el año 2017. Recuperado de: https://scielo.conicyt.cl/pdf/rci/v36n2/07161018-rci-36-02-0221.pdf

Ministerio de Salud. (2018). Plan nacional de prevención y control del VIH/SIDA e ITS. Recuperado de https://diprece.minsal.cl/wpcontent/uploads/2019/06/2019.06.12_PLANNACIONAL-VIH-SIDA-E-ITS.pdf

Mittal, M., Senn, T. E., \& Carey, M. P. (2012). Intimate partner violence and condom use among women: Does the informationmotivation-behavioral skills model explain sexual risk behavior?.AIDS and Behavior, 16(4), 1011-1019.

Ordoñez Sánchez, J., Real Cotto, J., Gallardo León, J., Alvarado Franco, H., \& Roby Arias, A. (2017). Conocimientos sobre salud sexual 
y su relación con el comportamiento sexual en estudiantes universitarios. In Anales de la Facultad de Medicina 78 (4),419-423. doi.org/10.15381/anales.v78i4.14264

Organización Mundial de la Salud. (2018). Directrices sobre encuestas bioconductuales en grupos de población con riesgo de VIH. Recuperado de: https://www.who.int/hiv/pub/guidelines/biobe havioral-hiv-survey/es/

Organización Mundial de la Salud. (2018). Informe resumido de la Reunión del grupo de trabajo de la OMS sobre el tratamiento del VIH en adultos sobre la optimización del tratamiento y el seguimiento del VIH, Boston, Estados Unidos. Recuperado de: https://www.who.int/hiv/pub/meetingreports/a rv-optimization-2018/en/

Paniagua, F. A., O'Boyle, M., Wagner, K. D., Ramirez, S. Z., Holmes, W. D., Nieto, J. F., \& Smith, E. M. (1994). AIDS-Related Items for Developing an AIDS Questionnaire for Children and Adolescents. Journal of Adolescent Research, 9(3), 311339. doi:10.1177/074355489493003

Pérez-Morente, M., A., Cano-Romero, E., Sánchez- Ocón, M., T., Castro-López, E., Jiménez-Bautista, F., \& Hueso- Montoro., C. (2017). Factores de riesgo relacionados con las infecciones de transmisión sexual. Revista Española de Salud Pública, 91,1-6.

Pineda, L. T. O., Rodríguez, A. F. U., \& Orbegozo, L. J. V. (2013). Conocimientos y actitudes frente al VIH/SIDA en padres de familia de adolescentes colombianos. Revista Colombiana de Psicología, 22(1), 59-73.

Pineda-Roa, C. A., \& Campo-Arias, A. (2018). Estudio psicométrico de una escala de actitud frente al VIH-sida en estudiantes de psicología de dos escuelas colombianas. Duazary: Revista Internacional de Ciencias de la Salud. 15(1), 51-59. doi.org/10.21676/2389783X.2022

Prata, N., Morris, L., Mazive, E., Vahidnia, F., \& Stehr, M. (2006). Relationship between HIV risk perception and condom use: Evidence from a population-based survey in Mozambique. International Family Planning Perspectives, 32(4), 192-200.
Programa Conjunto de las Naciones Unidas sobreel VIH/SIDA (2018). UNAIDS, DATA $2018 . \quad$ Recuperado de: https://www.unaids.org/en/resources/documen ts/2018/unaids-data-2018

Programa Conjunto de las Naciones Unidas sobreel VIH/SIDA (2019). UNAIDS data $2019 . \quad$ Recuperado de: https://www.unaids.org/en/resources/documen ts/2019/2019-UNAIDS-data

Programa Conjunto de las Naciones Unidas sobreel VIH/SIDA (2020). El informe de ONUSIDA sobre la epidemia mundial de sida muestra que los objetivos de 2020 no se cumplirán debido a un éxito profundamente desigual; COVID-19 arriesga desviar los avances en VIH fuera de curso. Recuperado de:

https://www.unaids.org/es/resources/presscent re/pressreleaseandstatementarchive/2020/july/ 20200706_global-aids-report

Rosenstock, I. M., Strecher, V. J., \& Becker, M. H. (1988). Social learning theory and the health belief model. Health Education Quarterly, 15(2), 175-183.

Santos, L. A. D., Couto, M. T., Mathias, A., \& Grangeiro, A. (2020). Hombres heterosexualmente activos, masculinidades, prevención de infección por VIH y búsqueda de profilaxis posexposición sexual consentida. Salud Colectiva, 15, 1-14. doi.org/10.18294/sc.2019.2144

Santos-Iglesias, P., Vallejo-Medina, P., \& Sierra, J. C. (2014). Equivalencia y baremos del Hurlbert Index of sexual assertiveness entre hombres y mujeres españoles. Anales de Psicología, 30(1), 232-237. doi:10.6018/analesps.30.1.143321

Schreiber, J. (2017). Update to core reporting practices in structural equation modeling. Research in Social and Administrative Pharmacy, 13(3), 634-643. doi.org/10.1016/j.sapharm.2016.06.006.

Semaan, S., Lauby, J., \& Liebman, J. (2002). Street and network sampling in evaluation studies of HIV risk-reduction interventions. AIDS rev, 4(4), 213-23.

Spencer, G., Doull, M., \& Shoveller, J. A. (2014). Examining the concept of choice in sexual health interventions for young people. Youth 
\& Society, $\quad 46(6), \quad 756-778$. doi.org/10.1177/0044118X12451277

Spindola, T., de Barro de Araujo, A. S., de Jesus Brochado, E., Fernanda Sousa Marinho, D., Rose Costa Martins, E., \& da Silva Pereira, T. (2020). Prácticas sexuales y comportamiento de jóvenes universitarios frente a la prevención de infecciones de transmisión sexual. Enfermería Global, 19(2), 109-140. doi.org/10.6018/eglobal.382061

Stutts, L. A., Robinson, P. A., Witt, B., \& Terrell, D. F. (2020). Lost in translation: College students' knowledge of HIV and PrEP in relation to their sexual health behaviors. Journal of American College Health, 1-7. doi:10.1080/07448481.2020.1757679

Tangarife, J. L., \& Arias, J. A. C. (2015). Construcción y validación de escalas de medición en salud: Revisión de propiedades psicométricassión de propiedades psicométricas. Archivos de Medicina, 11(3), 1.

Ugalde, C., Benadof, C., \& González, E. (2020). Human Immunodeficiency Virus: Attitudes and knowledge among health professionals in Santiago, Chile. Odontología Vital, (32), 3944.

Velo-Higueras, C., Cuéllar-Flores, I., Sainz-Costa, T., Navarro-Gómez, M. L., García-Navarro, C., Fernández-McPhee, C., ... GonzálezTomé, M. I. (2018). Jóvenes y VIH. Conocimiento y conductas de riesgo de un grupo residente en España. Enfermedades Infecciosas y Microbiología Clínica. doi:10.1016/j.eimc.2018.05.015

Villar-Loubet, O. M., Cook, R., Chakhtoura, N., Peltzer, K., Weiss, S. M., Shikwane, M. E., \& Jones, D. L. (2013). HIV knowledge and sexual risk behavior among pregnant couples in South Africa: The PartnerPlus project. AIDS and Behavior, 17(2), 479-487. doi:10.1007/s10461-012-0360-5.

Villaseñor-Sierra, A., Caballero-Hoyos, R., Martín, H. S., \& Santos-Preciado, J. I. (2003). Conocimiento objetivo y subjetivo sobre el VIH/SIDA como predictor del uso de condón en adolescentes. Salud Pública de México, 45(S1), 73-80.

De Wilde, M., Casini, A., Wollast, R., \& Demoulin, S. (2020). Sex is power belief and women's mental health: The mediating roles of self-objectification and sexual subjectivity. European Journal of Social Psychology, 50(5), 1017-1031. doi.org/10.1002/ejsp.2643

Zetterqvist, M., Svedin, C. G., Fredlund, C., Priebe, G., Wadsby, M., \& Jonsson, L. S. (2018). Self-reported nonsuicidal self-injury (NSSI) and sex as self-injury (SASI): Relationship to abuse, risk behaviors, trauma symptoms, self-esteem and attachment. Psychiatry Research, 26(5), 309316. doi.org/10.1016/j.psychres.2018.05.013 